\title{
The Lamellar Corneal Graft for Optical Indications
}

\author{
P. J. McDONNELL and M. G. FALCON
}

London

\begin{abstract}
Summary
The results of thirteen lamellar corneal grafts performed for optical indications are presented. Ten of the eyes maintained a clear graft; six patients achieved an acuity of $6 / 9$ or better.
\end{abstract}

The lamellar corneal graft for visual improvement was first performed during the last century ${ }^{(1)}$ and was a common procedure in the early and middle parts of this century when reasonable visual results were obtained. (2) In comparison the penetrating corneal graft was initially less successful because of problems with rejection and donor material quality. However the more recent ready availability of high quality donor corneas, the understanding and successful treatment of the rejection process, the appreciation of the importance of the endothelium, and advances in microsurgical technique have all helped to make penetrating keratoplasty a highly successful surgical procedure. For these reasons the majority of corneal transplants performed today are penetrating grafts.

Nevertheless the lamellar graft still offers some important advantages: the procedure is not intraocular, the patient's own endothelium is left intact and there is less risk of rejection. Modern microsurgical techniques have also improved the ease with which a lamellar graft may be performed. In patients with anterior or mid-stromal opacities and a normal endothelium a lamellar graft may be indicated in preference to a penetrating keratoplasty.

In recent years little has been published on the visual results of lamellar keratoplasty and there has been a tendency to overlook this procedure when considering patients for corneal graft surgery. We therefore reviewed a group of patients who had been selected for a lamellar graft for visual purposes at St. Thomas' Hospital since 1981.

\section{Materials and Methods}

The records of patients who had undergone a corneal graft at St. Thomas' Hospital since 1981 were reviewed and those cases of lamellar graft were identified. Details were taken from the case notes and the patients were reviewed in the clinic. The most recent patients were seen both pre- and postoperatively after the start of the study. All patients had been assessed preoperatively in the External Diseases Clinic, St. Thomas' Hospital. All surgery was performed in a similar fashion under general anaesthesia by either MGF or PJMcD. The trephine size was selected after assessment of the extent of the patient's corneal opacity. The trephine was used to mark the corneal epithelium to show the area of planned lamellar dissection. The superior right hand part of the trephine mark was then deepened vertically with a Beaver blade no. 7515 to the level of the planned dissection.

The tip of the blade was used to form an initial horizontal slit in the corneal stroma at the required level: the level was chosen with

Correspondence to: Mr. P. J. McDonnell, FRCS, South Wing Eye Department, St. Thomas' Hospital, London, SE1 7EH. 
the aim of removing all the stromal opacity in a single dissection and thus avoiding the need to repeat the procedure to remove a further layer of opacity.

This split in the stroma was extended with a Paufique's knife across the whole area outlined by the trephine mark, while the vertical edge of the disc was extended with a razor blade. Once separated the disc was left in position to protect the interface while the donor dissection was carried out. The donor lamella was prepared from either a whole eye or from a corneoscleral disc stored in organ culture medium. The intact globe was mounted on a whole eye stand, and the corneoscleral disc was mounted on a Baltimore stand which kept the disc fixed and provided an artificial anterior chamber of McCarey-Kaufman medium. A small vertical partial thickness incision was made in the peripheral cornea adjacent to the limbus with a Beaver blade no. 7515; the blade was used to start a horizontal split in the stroma at the appropriate depth to give the required thickness of lamella. This split was then further extended with a Paufique's knife, until it was possible to insert an iris repositor in the plane of cleavage. Further blunt dissection was carried out with a sweeping movement of the iris repositor until the plane of cleavage extended out to the limbus all round the cornea.

A trephine of the same size used on the recipient cornea was used to cut out the donor lamella. The donor lamella was sutured onto the recipient cornea, using two temporary stay sutures and a continuous suture, both of 10/0 nylon. At the end of surgery sub-conjunctival injections of cefuroxime $100 \mathrm{mg}$ and betamethasone $4 \mathrm{mg}$ were given.

\section{Results}

\section{Surgery}

Eleven patients who had undergone a lamellar graft at St. Thomas' Hospital since 1981 were identified (Table I). One of these patients had a repeat lamellar graft in one eye, and one patient had a lamellar graft in each eye, making a total of thirteen grafts in the study. During the same period there were a total of 132 patients in our unit who had had some form of corneal transplant surgery; patients undergoing lamellar grafts therefore accounted for $8 \%$ of the total.

Follow-up varied from a minimum of three months to a maximum of seven years, with a mean of 45.9 months. Age at the time of surgery ranged from 27 to 77 years, with a mean of 43.2 years. There were 9 males and 2 females in the study.

There was a range of corneal diseases treated with lamellar graft: pterygium (3 grafts), opacity from alkali burns (3 grafts), Salzmann's dystrophy (2 grafts), herpes simplex (2 grafts), and one graft each for macular dystrophy, intra-corneal haemorrhage, and adenovirus stromal opacities.

The size of graft ranged from $6.5 \mathrm{~mm}$ to

Table I Patient details

\begin{tabular}{|c|c|c|c|c|}
\hline Case & Diagnosis & $\begin{array}{l}\text { Follow up } \\
\text { time (mths) }\end{array}$ & Age & Sex \\
\hline 1 & macular dystrophy & 84 & 28 & $\mathrm{~m}$ \\
\hline 2 & alkali burn & 84 & 30 & $\mathrm{~m}$ \\
\hline 3 & intracorneal haemorrhage & 66 & 70 & $\mathrm{~m}$ \\
\hline 4 (2 grafts) & alkali burn & 64 & 29 & $\mathrm{~m}$ \\
\hline 5 & herpes simplex & 63 & 50 & $\mathrm{~m}$ \\
\hline 6 & pterygium & 49 & 27 & $\mathrm{~m}$ \\
\hline 7 & pterygium & 36 & 55 & f \\
\hline 8 & adenovirus opacities & 26 & 31 & f \\
\hline 9 & herpes simplex & 14 & 32 & $\mathrm{~m}$ \\
\hline $10(1)$ & Salzmann's dystrophy & 13 & 77 & $\mathrm{~m}$ \\
\hline $10(\mathrm{r})$ & Salzmann's dystrophy & 3 & 77 & $\mathrm{~m}$ \\
\hline 11 & pterygium & 6 & 46 & $\mathrm{~m}$ \\
\hline
\end{tabular}


$8.0 \mathrm{~mm}$ in diameter, with 5 grafts of $7.0 \mathrm{~mm}$ and 5 grafts of $7.5 \mathrm{~mm}$; three of the grafts used for patients with pterygium were of the keyhole type.

Ten of the thirteen grafts remained clear and six patients achieved $6 / 9$ or better. Figure 1 shows the best corrected visual acuity before and after surgery. Table II shows the relationship between best post-operative visual acuity and the diagnosis for each patient.

\section{Complications}

During surgery two patients had haemorrhage into the interface: in one patient the blood was easily cleared by irrigating the interface with balanced salt solution, and in the other the blood persisted into the post operative period but eventually cleared after three months. A microperforation occurred during preDescemet's deep lamellar dissection in one patient but as there was only a minimal leak the perforation was not repaired; the lamellar graft was sutured into place and the post operative course was uneventful.

One patient needed two lamellar dissections during surgery as some deep stromal opacity was still visible after the first dissection. Only one patient developed a presumed corneal graft rejection episode, which resolved rapidly after treatment with topical steroids. One patient had a transient rise in intraocular pressure which resolved when the post operative topical steroids were tailed off. Three patients had central epithelial defects. Patient No. 2, who had had a severe alkali burn years earlier, and

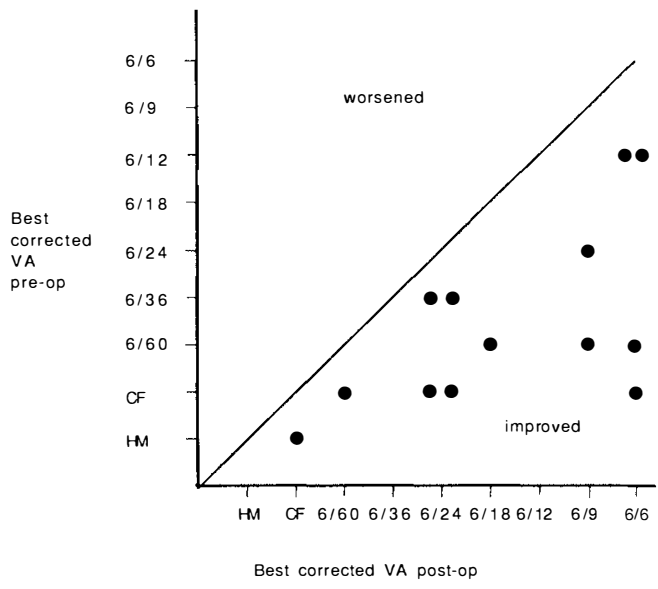

Fig. 1. Graph comparing best corrected preoperative with best corrected post-operative visual acuity in each eye receiving a graft. All eyes showed improvement in acuity.

Table II Visual results related to original diagnosis

\begin{tabular}{lll}
\hline $\begin{array}{l}\text { Best post-op. } \\
\text { corrected acuity }\end{array}$ & Case no. & Diagnosis \\
\hline $6 / 6$ & 1 & $\begin{array}{l}\text { macular dystrophy } \\
\text { intra-corneal haemorrhage } \\
\text { pterygium }\end{array}$ \\
& 3 & Salzmann's dystrophy \\
& 6 & pterygium \\
$6 / 9$ & $70(1)$ & adenovirus opacities \\
& 7 & alkali burn \\
$6 / 18$ & 8 & alkali burn \\
$6 / 24$ & $4(2 \mathrm{nd}$ graft $)$ & herpes simplex \\
& $4(1 \mathrm{st}$ graft $)$ & herpes simplex \\
& 5 & Salzmann's dystrophy \\
$6 / 60$ & 9 & pterygium \\
$3 / 60$ & $10(\mathrm{r})$ & alkali burn
\end{tabular}


previous surgery, developed a persistent epithelial defect which failed to respond to treatment over a period of a year and eventually perforated and needed a penetrating graft. Patient No. 4, who had also had an alkali burn, developed a central epithelial defect in association with active vascularisation of the graft host junction about one month after his first graft, having repeatedly failed to attend for his postoperative visits. The vascularisation and epithelial defect resolved after intensive topical steroids. The graft in the right eye of patient No. 10, who had Salzmann's dystrophy, developed a small epithelial defect two weeks post surgery which then healed over a period of six weeks with topical treatment only. Three grafts failed to stay clear: these were the graft in patient No. 2 (see above) and the two grafts in patient No. 4 which both eventually became vascularised and opaque. Four of the ten clear grafts gave a best post operative acuity of less than 6/9; in one patient this was due to cataract (No. 10 right), and in three patients it was due to astigmatism.

One of these three patients was pleased with his vision and did not want to try a contact lens and the other two patients are in the process of being fitted with contact lenses and will hopefully show a further improvement in acuity.

\section{Discussion}

Although our series of thirteen grafts is too small to allow statistical analysis it does provide some useful information about the indications and results for this surgical procedure, since there is little in recent literature concerning the visual results of lamellar grafting.

In 1976 a review $^{(3)}$ of corneal buttons submitted in the past to a pathology laboratory revealed an incidence of lamellar grafts in the late 1950 's of $28.9 \%$ falling to $3.2 \%$ of all grafts in the early 1970 's. In our series lamellar grafts represent about $8 \%$ of all corneal grafts performed. This higher figure in our series may simply be related to our willingness to consider this form of surgery where indicated.

The indications for optical lamellar graft have been considered by a number of authors. Casey and Mayer ${ }^{(4)}$ listed ReisBuckler's dystrophy, Salzmann's dystrophy, Hurler's syndrome, band shaped keratopathy, spheroid degeneration, and traumatic scars as the commonest indications.

Arentsen $^{(5)}$, and Paton ${ }^{(6)}$ both write in more general terms of any anterior nonvascularised corneal opacification when restoration of normal visual acuity is not of primary importance. Arentsen cites Salzmann's dystrophy as a typical example. Paton also refers to the management of keratoconus with lamellar keratoplasty. Both authors mention that the lamellar graft should be particularly considered in paediatric cases as penetrating grafts do less well in the young.

In our series all the patients had opacities limited to the anterior or mid stroma and appeared to have a normal endothelium. The series included patients with diagnoses similar to that mentioned by other authors such as Salzmann's dystrophy and pterygium. Our study also included two patients with stromal opacity due to alkali burns and two patients with opacities due to old herpes simplex disease. These patients were considered suitable for lamellar graft as the opacity was anterior and there were no signs of active inflammation or vascularisation.

Three recent reviews ${ }^{(4,5,6)}$ of the technique of lamellar grafting give no detailed evidence on visual results when the graft is performed for optical purposes alone. One of these reviews $^{(4)}$ states that "the visual acuity obtained is rarely better than 6/12". Our study suggests it is possible to get much better results than this. Six of the thirteen grafts performed resulted in a visual acuity of $6 / 9$ or better, and ten patients had clear grafts with a permanent improvement of vision.

The complications encountered in our series were similar to those mentioned by other authors. Our one patient who had an episode of rejection responded quickly to treatment. The prognosis for an allograft rejection in the cornea with a lamellar graft is better than a penetrating graft as the endothelium is not involved in the rejection process. 
The two patients with opacities due to alkali burns developed problems of persistent epithelial defect and graft opacity despite having inactive anterior stromal scars with minimal vascularisation and a reasonable tear film pre-operatively. Both patients failed many of their post-operative follow-up appointments (patient No. 4 spent frequent periods in prison) so this may have influenced the ultimate prognosis of the grafts.

This study has shown that the visual prognosis of the lamellar corneal graft for optical purposes can be better than is often quoted. Although technically more difficult, the surgery has fewer serious complications than penetrating keratoplasty, and the particular benefit of not being an intraocular procedure. We believe that in carefully selected patients the lamellar graft should still be the procedure of choice.

\section{References}

${ }^{1}$ von Hippel A: Eine neue Methode der Hornhauttransplantation. von Graefes Arch Ophthalmol 1888, 34: 108-30.

2 Paufique L, Sourdille G-P, Offret G: Les Greffes de la Cornee. Paris, Masson 1948.

${ }^{3}$ Arentsen JJ, Morgan B, Green WR: Changing indications for keratoplasty. $A m \quad J$ Ophthalmol 1976, 81: 313-8.

${ }^{4}$ Casey TA and Mayer DJ: Corneal Grafting, Principles and Practice. London, WB Saunders 1984.

${ }^{5}$ Arentsen JJ: Lamellar grafting. In: Corneal Surgery: Theory, Technique, and Tissue. Ed. Brightbill FS. St. Louis, CV Mosby 1986.

${ }^{6}$ Paton D: Lamellar keratoplasty. In: Symposium on medical and surgical disease of the cornea, Transactions of the New Orleans Academy of Ophthalmology, St. Louis, CV Mosby 1980. 